\title{
PRODUÇÃO DE 1,3-PROPANODIOL A PARTIR DE GLICERINA BRUTA POR CLOSTRIDIUM BUTYRICUM
}

\author{
V. da S. SAAB ${ }^{1}$, T. F. FERREIRA ${ }^{2}$, F. F. MARTINS ${ }^{3}$, C. M. S. RIBEIRO ${ }^{4}$, M. A. Z. COELHO \\ ${ }^{1}$ Instituto Federal do Rio de Janeiro, Departamento de Processos Químicos \\ ${ }^{2}$ Instituto Federal do Rio de Janeiro, Departamento de Processos Químicos \\ ${ }^{3}$ Universidade Federal do Rio de Janeiro, Departamento de Engenharia Bioquímica \\ ${ }^{4}$ Centro de Pesquisa Leopoldo Américo Miguez de Mello, Gerência de Biotecnologia \\ ${ }^{5}$ Universidade Federal do Rio de Janeiro, Departamento de Engenharia Bioquímica \\ E-mail para contato: nessa.saab@gmail.com
}

\begin{abstract}
RESUMO - Com a crescente produção de biodiesel, grandes quantidades de glicerina estão sendo injetadas no mercado, baixando expressivamente seus preços. Em geral, a glicerina tem se mostrado uma matéria-prima importante no setor biotecnológico, pois vem sendo empregada como a principal fonte de carbono em alguns processos fermentativos. Dessa forma, é possível utilizar a glicerina bruta na obtenção de um produto de interesse industrial, o 1,3-propanodiol (1,3-PDO), utilizando o microorganismo Clostridium butyricum. O 1,3-propanodiol é uma molécula orgânica bifuncional que possui grande aplicação nas reações de síntese de poliuretanos, poliésteres e poliéteres. O presente trabalho visou a produção de 1,3-PDO a partir de glicerina bruta por Clostridium butyricum. A cepa estudada apresentou grande capacidade de conversão de glicerina em 1,3-PDO, atingindo o rendimento de $0,56 \mathrm{~g} \cdot \mathrm{g}^{-1}$ com a produtividade de 1,37 g. $L^{-1} \cdot h^{-1}$ de $1,3-P D O$.
\end{abstract}

\section{INTRODUÇÃO}

O1,3-propanodiol (1,3-PDO) é um composto orgânico de caráter bifuncional que possui grande potencial de ser utilizado em várias reações de síntese como monômero. É comumente usado em policondensações para produzir poliésteres, poliuretanos e poliéteres. Ele também apresenta uma vasta gama de aplicações já que oferece propriedades melhoradas para solventes, adesivos, laminados, resinas, detergentes e cosméticos (Ferreira et al., 2012).

Os principais micro-organismos normalmente utilizados para produção de 1,3-PDO são Klebsiellapneumoniae, Citrobacterfreundii, Clostridium pasteurianum e Clostridium butyricum (Saxenaet al., 2009). O principal substrato que é utilizado nesta rota biotecnológica de produção de 1,3-PDO é o glicerol. Durante o processo o glicerol é desidratado para 3-hidroxipropionaldeído (3HPA) pelo glicerol desidratase. O produto da reação de desidratação, 3-HPA é reduzido a 1,3-PDO

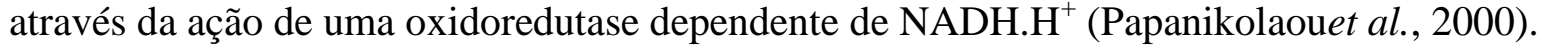


O aumento da produção de biodiesel propiciou uma maior oferta de glicerol no mercado, pois o glicerol também é obtido através da transesterificação alcalina que dá origem ao biodiesel. Dessa forma, a conversão microbiana de glicerol em 1,3-PDO é uma alternativa que permite agregar valor ao subproduto da produção de biodiesel, arcando de certa forma com o custo desse óleo tão importante atualmente no cenário mundial.

A literatura relata a produção de 1,3-PDO usando Clostridium butyricum, mas não há relatos sobre a utilização da estirpe Clostridium butyricum NCIMB 8082 para a produção de 1,3-PDO. Assim, o presente trabalho teve por objetivo avaliar a capacidade de crescimento da estirpe Clostridium butyricum NCIMB 8082 em glicerol bruto e a produção de 1,3-PDO.

\section{MATERIAIS E MÉTODOS}

\subsection{Cepa e Condições de Cultivo}

Foi utilizada a cepa Clostridium butyricum NCIMB 8082 através da NationalCollectionof Industrial, Foodand Marine Bacteria (NCIMB). O meio utilizado para reativação da cultura foi o ReinforcedClostridiaMedium e o Meio Fluido de Tioglicolato. O glicerol bruto foi obtido a partir de uma planta piloto de geração de biodiesel da Petróleo Brasileiro S/A (Petrobras).

\subsection{Metodologia Experimental}

Experimentos em frascos de vidro do tipo $\mathrm{SCHOTT}^{\circledR}$ : A cepa crescida foi mantida em frascos de penicilina, a $4^{\circ} \mathrm{C}$ contendo $50 \mathrm{~mL}$ do ReinforcedClostridiaMedium. Para inoculação, os frascos de penicilina contendo as células cultivadas foram incubadas a $37^{\circ} \mathrm{C}$ durante 30 minutos em agitador rotatório a $250 \mathrm{rpm}$. Depois disso, as células foram inoculadas em frascos de vidro do tipo SCHOTT ${ }^{\circledR}$ contendo 200 mLde meio. Foram utilizados dois diferentes meios que se encontram descritos na Tabela 1. Em ambos os meios foi purgado $\mathrm{N}_{2}$ antes e depois da inoculação. As amostras foram obtidas ao longo do tempo para analisar o consumo de glicerol, os produtos formados e o crescimento das células.

Tabela 1 - Composição dos diferentes meios utilizados.

\begin{tabular}{|c|c|}
\hline Meio 1 (Chatzifragkouet al., 2011) & Meio 2 (Wilkenset al., 2012) \\
\hline 3,4 g.L ${ }^{-1} \mathrm{~K}_{2} \mathrm{HPO}_{4}$ & \\
\hline 1,3 g.L. ${ }^{-1} \mathrm{KH}_{2} \mathrm{PO}_{4}$ & 2,71 g. $\mathrm{L}^{-1} \mathrm{KH}_{2} \mathrm{PO}_{4}$ \\
\hline 2,0 g.L ${ }^{-1}\left(\mathrm{NH}_{4}\right)_{2} \mathrm{SO}_{4}$ & 2,69 g. $\mathrm{L}^{-1} \mathrm{NH}_{4} \mathrm{Cl}-$ \\
\hline 0,2 g. $\mathrm{L}^{-1} \mathrm{MgSO}_{4} \cdot 7 \mathrm{H}_{2} \mathrm{O}$ & 0,186 g. $\mathrm{L}^{-1} \mathrm{MgSO}_{4} \cdot 7 \mathrm{H}_{2} \mathrm{O}$ \\
\hline 0,02 g. $\mathrm{L}^{-1} \mathrm{CaCl}_{2} \cdot 2 \mathrm{H}_{2} \mathrm{O}$ & 0,025 g. $\mathrm{L}^{-1} \mathrm{CaSO}_{4} \cdot 2 \mathrm{H}_{2} \mathrm{O}$ \\
\hline $1,0 \mathrm{~mL} \cdot \mathrm{L}^{-1} \mathrm{Fe}$ solution ${ }^{2}$ & 0,015 g. $\mathrm{L}^{-1} \mathrm{FeSO}_{4} \cdot 7 \mathrm{H}_{2} \mathrm{O}$ \\
\hline
\end{tabular}




\begin{tabular}{|c|c|}
\hline 1,0 g. $\mathrm{L}^{-1}$ extrato de levedura & 5,0 g.L $\mathrm{L}^{-1}$ extrato de levedura \\
\hline 20,0 g.L $\mathrm{L}^{-1}$ glicerina & 20,0 g. $\mathrm{L}^{-1}$ glicerina \\
\hline $2,0 \mathrm{~mL} \cdot \mathrm{L}^{-1}$ solução traço 1 & 3,0 mL.L-' ${ }^{1}$ solução traço 3 \\
\hline 2,0 g. $\mathrm{L}^{-1} \mathrm{CaCO}_{3}$ & $\mathrm{pH}=7,0$ \\
\hline $\mathrm{pH}=7,0$ & \\
\hline
\end{tabular}

$170 \mathrm{mg} . \mathrm{L}^{-1} \mathrm{ZnCl}_{2} ; 0,1 \mathrm{~g} . \mathrm{L}^{-1} \mathrm{MnCl}_{2} \cdot 4 \mathrm{H}_{2} \mathrm{O} ; 60 \mathrm{mg} \cdot \mathrm{L}^{-1} \mathrm{H}_{3} \mathrm{BO}_{3} ; 0,2 \mathrm{~g} \cdot \mathrm{L}^{-1} \mathrm{CoCl}_{2} \cdot 2 \mathrm{H}_{2} \mathrm{O} ; 20 \mathrm{mg} \cdot \mathrm{L}^{-1} \mathrm{CuCl}_{2} \cdot 2 \mathrm{H}_{2} \mathrm{O} ; 25 \mathrm{mg} \cdot \mathrm{L}^{-1}$ $\mathrm{NiCl}_{2} \cdot 4 \mathrm{H}_{2} \mathrm{O} ; 35 \mathrm{mg} . \mathrm{L}^{-1} \mathrm{Na}_{2} \mathrm{MO}_{4} \cdot 2 \mathrm{H}_{2} \mathrm{O} ; 0,9 \mathrm{~mL} . \mathrm{L}^{-1} \mathrm{HCl} 37 \%$.

25,0 g.L. $\mathrm{L}^{-1} \mathrm{FeSO}_{4} \cdot 7 \mathrm{H}_{2} \mathrm{O} ; 0,9 \mathrm{~mL} \cdot \mathrm{L}^{-1} \mathrm{HCl} 37 \%$.

${ }^{3} 1,0$ g.L $\mathrm{L}^{-1} \mathrm{FeCl}_{3} \cdot 4 \mathrm{H}_{2} \mathrm{O} ; 0,5$ g.L $\mathrm{L}^{-1} \mathrm{MnCl}_{2} \cdot 4 \mathrm{H}_{2} \mathrm{O} ; 0,85$ g.L $\mathrm{L}^{-1} \mathrm{CoCl}_{2} \cdot 6 \mathrm{H}_{2} \mathrm{O} ; 0,5$ g.L $\mathrm{L}^{-1} \mathrm{CaCl}_{2} \cdot 2 \mathrm{H}_{2} \mathrm{O} ; 0,5$ g.. $\mathrm{L}^{-1} \mathrm{ZnCl}_{2} ; 0,1$ g.L $\mathrm{L}^{-1}$ $\mathrm{CuCl}_{2} ; 0,05$ g.L $\mathrm{L}^{-1} \mathrm{H}_{3} \mathrm{BO}_{3} ; 0,05$ g.L.- $\mathrm{Na}_{2} \mathrm{MO}_{4} \cdot 2 \mathrm{H}_{2} \mathrm{O} ; 0,13$ g.L $\mathrm{L}^{-1} \mathrm{NiCl}_{2} \cdot 6 \mathrm{H}_{2} \mathrm{O} ; 5,0$ g.L $\mathrm{L}^{-1} \mathrm{NaCl} ; 0,1$ g.L. $\mathrm{L}_{2}^{-1} \mathrm{NaO}_{3} \cdot 5 \mathrm{H}_{2} \mathrm{O}$

Experimentos em Biorreator: A cepa crescida foi mantida em frascos de penicilina, a $4^{\circ} \mathrm{C}$ contendo $50 \mathrm{~mL}$ de ReinforcedClostridiaMedium. Os frascos de penicilina contendo as células cultivadas foram incubadas a $37{ }^{\circ} \mathrm{C}$ durante 30 minutos em agitador rotatório a $250 \mathrm{rpm}$ e depois inoculados num frascoSCHOTT ${ }^{\circledR}$ contendo $200 \mathrm{~mL}$ do Meio 1 . Após 18 horas, todo o meio de cultivo contido nos frascos foi transferido para um biorreator contendo $800 \mathrm{~mL}$ do Meio 1. Este procedimento foi realizado com purga de $\mathrm{N}_{2}$. Amostras foram obtidas ao longo do tempo para analisar o consumo de glicerol, os produtos formados e o crescimento celular.

O experimento foi realizado sob condições anaeróbias em biorreator com capacidade útil de $1 \mathrm{~L}$, a 37 ${ }^{\circ} \mathrm{C}$ e agitação de $200 \mathrm{rpm}$ durante 30 horas, com controle de temperatura e de $\mathrm{pH}$. O meio de alimentação foi composto por glicerol $\left(200 \mathrm{~g} . \mathrm{L}^{-1}\right)$ e extrato de levedura $\left(10 \mathrm{~g} . \mathrm{L}^{-1}\right)$.

\subsection{Métodos Analíticos}

Determinação do Crescimento Celular: O crescimento foi acompanhado através da leitura de densidade ótica (D.O.) a $600 \mathrm{~nm}$.

Análise:Para quantificar1,3-propanodiol e os subprodutos da fermentação: butanol, 2,3butanodiol, etanol e alguns ácidos orgânicos (butírico, succínico, cítrico, pirúvico, láctico e acético) foram realizadas análises de Cromatografia Líquida de Alta Eficiência (CLAE). Para tal análise utilizou-se os seguintes itens: coluna Aminex ${ }^{\circledR}$ HPX-87H IonExclusion de dimensões $300 \mathrm{~nm}$ x 7,8 nm (Bio-RadLaboratories), detector de índice de refração RID-10A (Shimadzu), bomba LC-20ADSP (Shimadzu) e software cromatográfico: LabSolutions (Shimadzu). A fase móvel utilizada foi $\mathrm{H}_{2} \mathrm{SO}_{4} 5$ $\mathrm{mM}$ com vazão de $0,8 \mathrm{~mL} / \mathrm{min}$, o volume de injeção foi $20 \mu \mathrm{L}$ e a temperatura da corrida $60^{\circ} \mathrm{C}$. Os padrões (Sigma-Aldrich) de todos os analitos estudados foram diluídos em água Mili-Q e injetados em triplicata para preparação da curva padrão, que relaciona a área obtida no cromatograma com a concentração do composto. As amostras foram filtradas em membrana (CHROMAFIL ${ }^{\circledR}$ ) com diâmetro de $0,45 \mu \mathrm{m}$ e injetadas em duplicata para a quantificação através do uso da curva padrão.Determinação do $\mathrm{pH}$ : $\mathrm{O} \mathrm{pH}$ inicial e final dos experimentos realizados em frascos do tipo SCHOTT $^{\circledR}$ foram analisados em um modelo de pH Digimed DM-22. 


\section{RESULTADOS E DISCUSSÃO}

Nos ensaio foi utilizado glicerol bruto contendo $80 \%$ de pureza e mesmo com as impurezas presentes no meio, a cepa foi capaz de consumir o substrato e crescer.A maior parte do glicerol foi consumido após 30 horas de fermentação, mesmo com o baixo inóculo utilizado, como mostra a Tabela 2. Os dados coletados mostram uma variação da concentração inicial do glicerol, este fato se dá pela dificuldade de amostragem causada por sua viscosidade.

Tabela 2 - Resultados obtidos nos experimentos em frascos SCHOTT ${ }^{\circledR}$ utilizando-se os meios 1 e 2.

\begin{tabular}{|c|c|c|c|c|c|c|c|c|c|c|}
\hline & \multicolumn{9}{|c|}{ Meio 1 } & \multicolumn{5}{c|}{ Meio 2 } \\
\hline $\begin{array}{c}\text { Tempo } \\
(\mathrm{h})\end{array}$ & $\begin{array}{c}\text { D.O. } \\
600 \mathrm{~nm}\end{array}$ & $\begin{array}{c}\text { Glicerol } \\
\left(\mathrm{g} . \mathrm{L}^{-1}\right)\end{array}$ & $\left.\begin{array}{c}\text { PDO } \\
\left(\mathrm{g} . \mathrm{L}^{-1}\right)\end{array}\right)$ & $\begin{array}{c}\text { Acetato } \\
\left(\mathrm{g} . \mathrm{L}^{-1}\right)\end{array}$ & $\begin{array}{c}\text { Succinato } \\
\left(\mathrm{g} . \mathrm{L}^{-1}\right)\end{array}$ & $\begin{array}{c}\text { D.O. } \\
600 \mathrm{~nm}\end{array}$ & $\begin{array}{c}\text { Glicerol } \\
\left(\mathrm{g} . \mathrm{L}^{-1}\right)\end{array}$ & $\begin{array}{c}\text { PDO } \\
\left(\mathrm{g} . \mathrm{L}^{-1}\right)\end{array}$ & $\begin{array}{c}\text { Acetato } \\
\left(\mathrm{g} . \mathrm{L}^{-1}\right)\end{array}$ & $\begin{array}{c}\text { Succinato } \\
\left(\mathrm{g} . \mathrm{L}^{-1}\right)\end{array}$ \\
\hline 0 & 0,367 & 16,3 & 0,00 & 0,00 & 0,00 & 0,261 & 19,0 & 0,00 & 0,00 & 0,00 \\
\hline 4 & 0,298 & 16,1 & 0,00 & 0,24 & 0,05 & 0,244 & 18,9 & 0,00 & 0,07 & 0,07 \\
\hline 8 & 0,275 & 11,3 & 1,05 & 1,40 & 0,43 & 0,198 & 17,9 & 0,41 & 0,26 & 0,26 \\
\hline 24 & 3,900 & 6,0 & 5,25 & 1,23 & 0,36 & 0,800 & 16,7 & 0,65 & 0,11 & 0,11 \\
\hline 28 & 3,960 & 5,6 & 5,37 & 1,21 & 0,37 & 1,300 & 15,2 & 2,57 & 0,06 & 0,06 \\
\hline 30 & 3,960 & 4,7 & 5,45 & 1,38 & 0,34 & 1,300 & 10,8 & 4,56 & 0,07 & 0,07 \\
\hline
\end{tabular}

Como observado na Tabela 2, o crescimento das células foi maior no Meio 1 do que no Meio 2 e o consumo do glicerol e a produção do 1,3-PDO também foram mais elevadas no Meio 1. É possível notar que, após 24 horas de fermentação, permaneceram 5,6 g.L $\mathrm{L}^{-1}$ do glicerol no Meio 1 e 16,7 g.L. $\mathrm{L}^{-1}$ no Meio 2. Ao mesmo tempo, a concentração de 1,3-PDO foi de 5,25 g.. ${ }^{-1}$ no Meio 1 e apenas 0,65 g.L ${ }^{1}$ no Meio 2. E ainda, após 30 horas de fermentação, a concentração de glicerol permanece em quantidade significativa. É provável que este fato ocorra devido a redução do $\mathrm{pH}$ que, possivelmente,inibe o metabolismo celular. $\mathrm{O}$ pH final dos meios foi de 4,66 e 4,69 para os meios $1 \mathrm{e}$ 2 , respectivamente, sendo menor no meio que apresentou a maior produção de acetato e succinato.

Apesar do Meio 2 ter fornecido um maior rendimento de glicerol em 1,3-PDO (56 \% g.g ${ }^{-1}$ ) em comparação com o Meio 1 (50 \% g.g $\left.{ }^{-1}\right)$, este último mostrou uma maior produtividade de 1,3-PDO $\left(0,33 \mathrm{~g} \cdot \mathrm{L}^{-1} \cdot \mathrm{h}^{-1}\right)$. A produtividade no Meio 2 foi de $0,21 \mathrm{~g} \cdot \mathrm{L}^{-1} \cdot \mathrm{h}^{-1}$ de $1,3-\mathrm{PDO}$. Assim, o Meio 1 foi escolhido para realizar o experimento em biorreator com controle de $\mathrm{pH}$ de modo a avaliar a capacidade da cepa de produzir o produto de interesse.

A Figura 1 apresenta os dados de crescimento celular, consumo de glicerol, produção de 1,3-PDO e $\mathrm{pH}$ do experimento realizado em biorreator. Mesmo iniciando o experimento com uma baixa densidade ótica, observa-se que, em poucas horas de experimento, foi obtida uma elevada produção de 1,3-PDO. É possível notar também que todo glicerol foi consumido em 6 horas, produzindo 9,40 g.. - $^{-1}$ de $1,3-P D O$.

Através de uma análise visual, observou que as células começaram a se aglomerar perto de seis horas 
de fermentação, formando grandes pelets. Provavelmente este fato ocorreu por causa da escassez de nutrientes. Depois disso, o biorreator foi alimentado com solução contendo glicerina e extrato de levedura e, em seguida, verificou-se o desaparecimento dos pelets, pois as células voltaram a se dispersar no meio.

Observa-se na Figura 1 que após a alimentação, oClostridium butyrium NCIMB 8082 foi capaz de continuar a consumir rapidamente o glicerol. Depois de um total de 10 horas de fermentação, a concentração de 1,3-propanodiol atingiu aproximadamente 14,0 g. $\mathrm{L}^{-1}$. A produtividade do experimento em biorreator foi de cerca de $1,4 \mathrm{~g} \cdot \mathrm{L}^{-1} \cdot \mathrm{h}^{-1}$ de 1,3 -PDO e o rendimento de glicerol em 1,3PDO foi de $0,56 \mathrm{~g} \cdot \mathrm{g}^{-1}$.

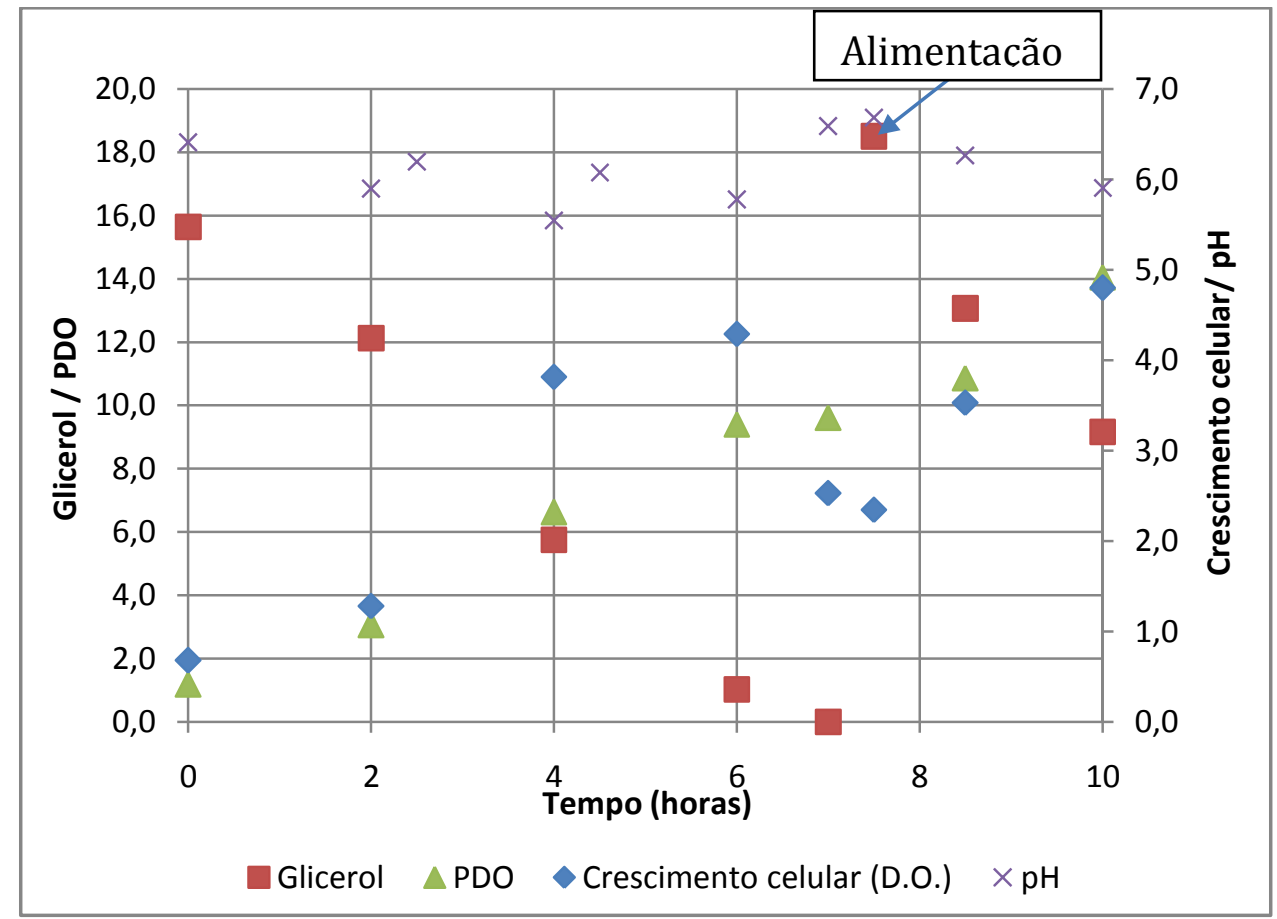

Figura 1 - Crescimento celular (D.O.), consumo de glicerol, produção de PDO e pH observados no experimento em biorreator com a cepa Clostridium butyricum.

O controle do pH é muito importante para melhorar a produção do 1,3-PDO. Bieblet al. (1999) produziu 1,3-PDO a partir de glicerol por Klebsiella pneumonia e observou o maior aumento de 1,3PDO em pH 7,0.

Chatzifragkouet al. (2011) realizaram a fermentação em batelada alimentada utilizando uma cepa de Clostridium butyricum. A concentração final de 1,3-PDO foi de 67,9 g. $\mathrm{L}^{-1}$ e o rendimento foi de 0,55 g. $\mathrm{g}^{-1}$. No entanto, estes resultados foram obtidos após 96 horas de fermentação, de modo que a produtividade foi de $0,70 \mathrm{~g} . \mathrm{L}^{-1} \cdot \mathrm{h}^{-1}$, inferior a obtida no presente trabalho usando a mesma composição 
de meio.

Wilkenset al. (2012) também realizaram batelada alimentada em biorreator de 1 L e obtiveram 76,2 g. $\mathrm{L}^{-1}$ de 1,3-PDO com uma produtividade de $2,3 \mathrm{~g} . \mathrm{L}^{-1} \cdot \mathrm{h}^{-1}$ após 32,5 horas de fermentação. No entanto, neste estudo os pesquisadores utilizaram que uma cepa deClostridium butyricum geneticamente modificada e um meio de cultura com mais nutrientes do que o usado no presente trabalho.

\section{CONCLUSÕES}

A cepa estudada se mostrou altamente eficaz na produção de 1,3-PDO, por se tratar de uma estirpe sem modificação genética, com boa produtividade e rendimento. Além disso, a mesma foi capaz de utilizar um resíduo industrial gerado em excesso no mercado brasileiro, o glicerol bruto, como fonte de carbono para seu crescimento. Na literatura podemos encontrar resultados satisfatórios de produção de 1,3 PDO porClostridium butyricum como os obtidos no presente trabalho, porém tratam-se de estirpes geneticamente modificadas, fato que dificulta a sua utilização em escala industrial, aumentando seus custos. Em contrapartida, a estirpe Clostridium butyricum NCIMB 8082 apresentou uma produtividade de $1,4 \mathrm{~g} \cdot \mathrm{L}^{-1} \cdot \mathrm{h}^{-1}$ e rendimento de $0,56 \mathrm{~g} \cdot \mathrm{g}^{-1}$ sem qualquer alteração genética e sem otimização das condições de produção. Vale ressaltar que a alimentação de glicerol foi realizada com 7,5 horas de fermentação e nesse momento o glicerol presente no meio de cultivo já havia se esgotado, sendo assim a cepa ficou um tempo sem substrato, o que provavelmente diminuiu a produtividade do processo.

Dessa forma, o presente trabalho mostra o potencial de produção de 1,3-PDO a partir de glicerina bruta utilizando o Clostridium butyricum NCIMB 8082, demonstrando que se trata de uma cepa potencial a ser estudada para esta finalidade.

\section{REFERÊNCIAS}

BIEBL, H.; MENZEL, K.; ZENG, A.P.; DECKWER, W.D. Microbial production of 1,3-propanodiol. Applied Microbiology and Biotechnology, v. 52, p. 289-297, 1999.

CHATZIFRAGKOU, A.; PAPANIKOLAOU, S.; DIETZ, D.; DOULGERAKI, A.I.; NYCHAS, G.J. E.; ZENG, A.P. Production of 1,3-propanediol by Clostridium butyricum growing on biodieselderived crude glycerol through a non-sterilized fermentation process Applied Microbiology and Biotechnology. 2011.

FERREIRA, T.F.; RIBEIRO, R.R.; RIBEIRO, C.M.S.; FREIRE, D.M.G.; COELHO, M.A.Z. Evaluation of 1,3-Propanediol Production from Crude Glycerol by Citrobacterfreundii ATCC 8090. Chemical Engineering Transactions, v. 27, p. 157-162, 2012.

PAPANIKOLAOU, S.; RUIZ-SANCHEZ, P.; PARISET, B.; BLANCHARD, F.; FICK, M. High 
production of 1,3-propanediol from industrial glycerol by a newly isolated Clostridium butyricum strain. Journal of Biotechnology, v. 77, p. 191-208, 2000.

SAXENA, R.K.; ANAND, P.; SARAN, S.; ISAR, J. Microbial production of 1,3-propanediol: recent development and emerging opportunities. Biotechnology Advances, v. 27(6), p. 895-913, 2009.

WILKENS, E.; RINGEL, A.K.; HORTIG, D.; WILLKE, T.; VORLOP, K.D. High-level production of 1,3-propanediol from crude glycerol by Clostridium butyricum AKR102a. AppliedMicrobiologyandBiotechnology, v. 93, p. 1057-1063, 2012. 